\title{
Cognitive Sustainability in the Age of Digital Culture
}

\author{
Luis Emilio Bruni
}

\author{
leb@media.aau.dk, Department of Architecture, Design and Media Technology, Aalborg University, \\ Denmark
}

\begin{abstract}
The aim of this article is to contextualize the implications of the expansion of digital culture in the on-going discussions about the relations between sustainability and information and communication technologies. In order to relate the development of a global digital communication web, its effects on cultural processes and the issues of ecosystem and human sustainability that humanity is facing, I will relate and elaborate on three aspects: 1) A Batesonean perspective on sustainability 2) The recent evolution of the technosphere, and 3) Yuri Lotman's notion of Semiosphere and his semiotic theory of culture. This path will lead me to delineate some of the eco-ethical dimensions implied in the development of pervasive digital-interactive-immersive-representational technologies.
\end{abstract}

Keywords: Biosphere, Technosphere, Semiosphere, sustainability, pervasive digital-interactive-immersive-representationaltechnologies

\section{Introduction}

In the present work I will overlap two clear and well acknowledged trends that have developed in the last half a century in globalized contemporary society:

- The advent of what we can denominate "digital culture", and

- The collective awareness of the so-called "ecological crisis", and the related notion of sustainability.

The objective of this exposition is to contextualize the impact of the expansion of digital culture in the on-going discussions about the relations between sustainability and information technology. This path will lead me to delineate some of the eco-ethical dimensions implied in the development and expansion of pervasive digital-interactive-immersive-representational technologies.

I will consider in particular the technological developments that are related to explicit multimodal representations supported by interactive and immersive digital technologies, trying to keep in focus the fate and the ecology of the "content" that can be mediated through such technological set-ups.

In this trend we can include established, as well as emerging, technological fields or paradigms such as ubiquitous and pervasive computing, ambient intelligence, wearable computing, virtual and augmented reality, affective computing, to name a few.

Most of these fields do not represent a kind of technology per se but a manifested intention of combining and integrating different kinds of technologies in particular directions. This convergence of many types of technologies and platforms is what has made possible the advent of digital culture. Globalization as we understand it today is embedded in this phenomenon of digital culture.

In order to relate the development of a global digital communication web, its effects on cultural processes and the issues of ecosystem and human sustainability that humanity is facing, I will define and elaborate on the following three aspects:

- A Batesonean perspective on sustainability

- The recent evolution of the technosphere

- Yuri Lotman's notion of Semiosphere and the implications of his semiotic theory of culture 


\section{The Three Positive Feedbacks of the Ecological Crisis}

Much has been said about the "ecological crisis" in the last 50 years and many great thinkers and scientists have agreed that the roots of the crisis lay at the cultural level (see for example Bateson (1972) and Jonas (1984)). Some scholars have also pointed out that "crisis thinking" may be counterproductive as a way to promote cultural and environmental responsibility (Mueller, 2009). In any case, it is not only the ecosystems that are in crisis (although they manifest the consequences of such crisis) but deep-rooted cultural values: "Epistemological error is often reinforced and therefore self-validating. You can get along all right in spite of the fact that you entertain at rather deep levels of the mind premises which are simply false ... circuits and balances of nature can only too easily get out of kilter, and they inevitably get out of kilter when certain basic errors of our thought become reinforced by thousands of cultural details." (Bateson, 1972)

In 1972, anthropologists and biologists Gregory Bateson, one of the founder figures of cybernetics, explained the origins of the "ecological crises" as the working of three main causes interacting and potentiating each other in a system of positive feedbacks (Bateson, 1972). These three main causes were in his view:

- Population increase

- Technological advance and innovation

- Errors in the thinking and attitudes of our culture, something that he characterized as "hubris", an old Greek notion that in Modern time has come to denote extreme arrogance and pride. By overestimating our capabilities, knowledge and power, we are thus prone to lose touch with reality and lose perspective of where things are going.

These three factors implied in the "crisis" were schematized by Bateson as shown in Figure 1. First of all, each factor works as a self-reinforcing feedback loop in which more population brings more population, new technology stimulates further innovation and technology dependence, and finally the collective hubris becomes reinforce and potentiated by thousands of cultural details, which are usually taken for granted or unconsciously assumed.

Similarly, all three factors reinforce each other in pairs, as for example, the more "effective" technologies we develop the more population and life expectancy increases, and vice versa, the more population increases the more technology is necessary to sustain it. Or, the more we are convinced that our science and technology will solve all our problems, the more technology and technological innovation will increase, and again, the more technology we have in our hands the more control we believe we have. The result is a large loop involving all three causes reinforcing each other and leading to environmental decay, hunger, social disarray and war.

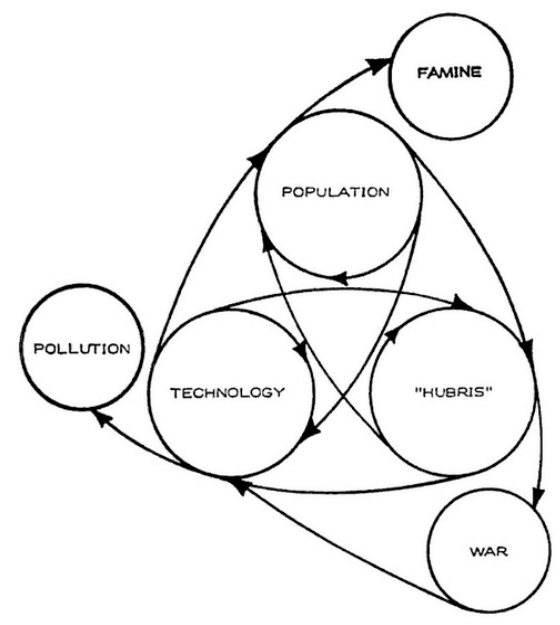

Figure 1: The three positive feedbacks of the ecological crisis as presented by Gregory Bateson in "Steps to an Ecology of Mind" (Bateson, 1972) 
According to Bateson, in order to head towards a more sustainable direction we should counter balance at least one of these three critical positive feedbacks. Among these three factors I will be concentrating in "technology" and "hubris", or in other words, the relation between technological evolution and the cultural process in contemporary global society (Figure 2).

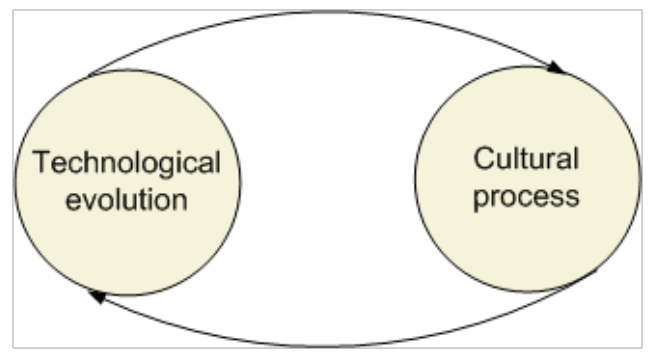

Figure 2: The mutual determination of technological evolution and cultural processes in contemporary society

It is worthwhile pointing out that Bateson does not propose to prevent further technological progress but he foresaw the possibility that it could be steered in appropriate directions. Seen in the new context of digital culture this would imply the use of technology for steering our cultural process in sustainable directions. In order to characterize technology and culture in a dynamic way, in the following sections I will relate them respectively to the notions of "technosphere" and "semiosphere".

\section{The Technosphere}

As a consequence of the Industrial Revolution the technosphere has grown exponentially driven by the close interrelation between science and technology. Particularly, with the advent of digital technology, the technosphere has been expanded not only geographically but it has also grown qualitatively in connectivity, giving rise to a global web of infrastructure for information management and communication sparse throughout the entire biosphere and even reaching out to the atmosphere into the already crowded geostationary orbits around the earth. This huge techno-web - which continues expanding and evolving in scope and ubiquitousness - can be said to be the vertebral column of what we perceive as globalization and of the rise of what we call "digital culture". Paradoxically, it is this interconnectivity through the technosphere which has contributed to the consolidation of the perception of the so called global issues and the unsustainability of the dominating cultural patterns.

It is hard to trace back the original coining of the term "technosphere". It is clear however that the concept is a derivative of Eduard Suess and Vladimir Verdnasky's complimentary concept of "biosphere" and Teillard de Chardin's notion of "noosphere". Because of the unusual formulation of the latter, the concept of technosphere sometimes appears in some contexts as lacking academic rigor. Nonetheless, it is properly and widely used in many scientific and academic fields as varied as Environmental Risk Assessment (see for example Blok (2005)), Industrial Ecology, Life Cycle Analysis and Ecology in general, usually referring to the built-environment, as oppose to the natural environment.

A very relevant early reference (although surely not the first one) can be found in Michel Batisse's article from 1973 "Environmental Problems and the Scientists". Batisse, an influential figure in the development of the United Nations international network of institutions and treaties for sustainability, put it this way:

We have seen that the problems of the environment, as they appear objectively, are found at the interface between the biosphere and the technosphere. The role of the natural scientists in their research work is precisely to study this interface, which implies on the one hand the 
structure and functioning of the biosphere under human pressure and, on the other hand, research on the modification of the technosphere in a direction less damaging to the environment (Batisse, 1973).

In the same article he introduced the notion of technosphere as follows:

Immediately above the biosphere, and now surrounding it entirely, is a higher level of organization, which has become important only recently, and which can be called the technosphere. This is not only made up of the factories, the dams and the irrigated fields, but also the whole canvas of technological facts and features of a physical, chemical or biological nature (Batisse, 1973).

The global satellite, wired and wireless, communication platform and infrastructure to which we are now so accustomed and through which our dynamic cultural exchanges have been incredibly accelerated, has its roots in previous institutional networks (military, scientific, political, economical) that were set out to implement and use such an enormous technological web to gather and communicate data, information and knowledge on local/global processes and to conform natural, military, economic, social, agricultural and infrastructure databases.

As known, this web has been growing through the proliferation of structures that include a great variety of artifacts, hardware, software, networks of monitoring and communication satellites and a great variety of remote-sensing techniques, data acquisition, manipulation and display through large computing and modeling, in situ sensors for advance site characterization and monitoring, artificial intelligence and decision making technology, information sharing and communication technology, etc.

The technological mutation implicit in our global monitoring, information and communication systems is the result of the development and integration of various technologies such as: remote and in situ sensing, data telecommunication, technology for the manipulation, representation and "intelligent" management of data, aerospace and military technology, among many others.

The increasing commercialization of this techno-web has implied a fusion with the great advances in new media technology and with the prospect of further developments into new forms of technological integration at local and global levels, extending its scope to all major sectors of contemporary society, including commerce, marketing, finances, advertisement and in general almost all aspects of social and cultural relations.

In the present framework, the notion of technosphere serves to outline a sketch of the constellation of paradigms and disciplines advocating for technological integration, expansion and connectivity of "digital interactive immersive and representational technologies". The customary denominations of certain sectors, such as "media technology" or "information and communication technology" seem to fall short in order to encompass the ever-increasing levels of integration proposed by the new tendencies. I will use here the denomination "digital-interactive-immersive-representationaltechnologies" (DIIRT) in order to include most of these developments, however keeping in mind that not all the functional integrated units and platforms necessarily include all these characteristics.

Among these trends we can mention for example ubiquitous and pervasive computing, ambient intelligence, virtual reality, affective computing, wearable computing, augmented reality, embedded network systems, tangible interfaces, seamless interfaces, nanotechnology and enactive interfaces, among many others.

These tendencies intend to create and implement innovative local ramifications and ubiquitous "points of entrance" (of commercial and civilian interest) to the global cultural-institutional technoweb already in place and in continuous evolution. Thus two levels or trends have been identified here, which are closely related to the implementation of strategies for sustainability through the technosphere:

- The hyper connected global society, which determines the boom of digital culture

- The institutional intergovernmental web of monitoring and information systems 
It is important to understand how this grand technological web is itself generated by, and in the same time lodges, a cognitive and cultural process with deep-rooted premises that may coincide in many cases with those that have originated the "crisis" in the first place.

\section{The Semiosphere}

In semiotics when one speaks about texts and languages there is a much broader connotation of these two terms than in our daily use of them. So a text can be any content supported by any transmission medium and implemented in a language appropriate to that medium - independent of whether such language is symbolic, iconic or a combination of both, and also independent of whether it is intended to unimodal or multimodal reception, if seen from the cognitive point of view.

In some sort of atomistic, and rather static, view, the "semiotic universe" could be regarded as the totality of individual texts and isolated languages as they relate to each other. In this case, says Lotman (2005/1984), all structures will look as if they are constructed out of individual bricks. According to him it is more useful to take a more organic and dynamic point of view: all semiotic space may be regarded as a unified system in a dynamic process. In this case, primacy does not lie in one or another sign of whatever language, but in the "greater system", namely the "Semiosphere". The semiosphere is the semiotic space, outside of which the whole of communication, i.e.: semiosis itself, cannot exist.

In his Semiotic Theory of Culture, Lotman (2005/1984; 1990), defines the Semiosphere as "the whole semiotic space of the culture in question". In analogy to Vernadsky's biosphere, the semiosphere is the necessary virtual semiotic space for the existence and functioning of all languages. It is a sort of emergent dynamic system (or process) that could not be seen as just the sum total of all past and existing different languages and cultural products (i.e. texts) that are and have been implemented in such languages.

This semiotic space, the semiosphere, is the necessary condition for culture to continue its historical unfolding. It has a diachronic dimension in the sense that through the use of languages and media we are in touch with the "virtual" facts (since they don't exist anymore) and narratives from the past and from many different cultural currents that have taken life throughout human history. It also simultaneously has a synchronic dimension implied by the active cultural process of the present where so many languages, cultural texts and currents mingle continuously in many dynamic layers of the whole semiosphere. The continuous diachronic-synchronic interplay determines the extension or the reach of a particular culture in a particular period. If we consider the current state of contemporary globalised culture we can claim that the evolution of the technosphere has enormously augmented the possibilities to navigate and reach synchronically and diachronically much larger extensions of the semiosphere than ever before. The hyper availability of texts and cultural representations of all kinds in real time expand the geography of human knowledge and cultural production as never before. Not only because of ease of access but also because of accumulated quantity. But more is not necessarily better, as a positivistic view of progress would claim. The semiosphere is a dynamic space were many small and big, past and present, "sub-semiospheres" continuously interact creating new layers of meanings, transforming information and knowledge by continuous translations and interpretations of the old and the new, of the official and the fringe, of what belongs to the center of a given sub-semiosphere and what comes from the periphery to conquer the center and transform its very nature.

One feature claimed by Lotman is the semiosphere's incredible heterogeneity. The languages that fill up the semiotic space are various and they relate to each other along a spectrum that may go from complete mutual translatability to complete mutual untranslatability. At all stages of development there are contacts with texts and cultural products coming in from cultures which formerly lay beyond the boundaries of the given semiosphere. Because of the impossibility of complete translatability these "cultural invasions", sometimes by whole cultural layers, affect the internal structure of the "world picture" of a given culture. These dynamic relations, superpositions and transformations get a critical and hypercomplex nature when it comes to "digital culture". Across 
any synchronic section of the semiosphere different languages, texts and cultural processes at different stages of development may be in conflict. For example, some texts may be immersed in languages not their own while the codes to decipher them may be entirely absent. The acceleration of these processes may have huge consequences for the tensions and trade-offs between cultural diversity and homogenization and for the emergence and proliferation of the thousands of unsustainable cultural details to which Bateson was referring. The concept of semiosphere brings cultural analysis into contact with the history of culture and with the newest phenomena in culture (Torop, 2005), including of course what interests us here, i.e.: "digital culture".

The virtual space of culture that we are calling, after Lotman, the semiosphere, has always counted with a physical substrate for its maintenance and actualization. In this regard the first cognitive technology that needs to be mentioned is natural language itself, both for synchronic and diachronic communication, starting with oral tradition. Even at this stage there is a physical substrate for the dynamic cultural exchange (in the semiosphere), in this case the network of human beings with their physiological bodies and cognitive systems. But there has also been all along the development of multimodal cognitive technologies to support the expansion of the semiosphere, also of highly iconic nature, as the long history of media illustrates, from cave paintings to augmented reality. Until we find ourselves in the present technospheric configuration of connectivity, pervasiveness and synchronicity in which new technologies have given rise to what we call "digital culture", due merely to an extension of the semiosphere's substrate, i.e. the technosphere - and both its synchronic and diachronic reach - allowed by the advent of digital technology.

The analytical semiosphere/technosphere dichotomy illustrates the more general considerations of the distinction, in the specific case of media, between "content" and "form". By referring to Lotman's dynamic cultural ecology we obtain a more dynamic model in order to better characterize the complexity of the issue. The "content" stored in the technosphere is on a continuous basis dynamically actualized and transformed by the interactive cognitive and semiotic process of millions of human agents. As eloquently put by neuroscientist Steven Rose: "With its hundred billion nerve cells, with their hundred trillion interconnections, the human brain is the most complex phenomenon in the known universe - always, of course, excepting the interaction of some six billion such brains and their owners within the socio-technological culture of our planetary ecosystem!" (Rose, 2005) to which we would like to add the further complexity given by the diachronic interaction of our current six billion brains with the many other billions that have preceded us.

\section{Towards an Intelligent Technosphere}

The prospect is that through ubiquitous computing, ambient intelligence and augmented reality we will be continuously "immersed" and have the possibility to interact in an "on-line" cultural process mediated by all kinds of new ways of representing our thoughts, emotions, ideas, beliefs, opinions and behaviors.

The substrate of the semiosphere is in this way expanded, and those who have access have the possibility to navigate into new frontiers of the semiosphere which were not possible before - for good or for bad.

Already in 1972 Gregory Bateson offered some hints on how to re-orient information technology along a more sustainable path and sketched a definition for a healthy ecology of human civilization:

It would be convenient to have an abstract idea of what we might mean by ecological health. Such a general notion should both guide the collection of data and guide the evaluation of observed trends. [...] A single system of environment combined with high human civilization in which the flexibility of the civilization shall match that of the environment to create an ongoing complex system, open-ended for slow change of even basic (hard-programmed) characteristics (Bateson, 1972).

Among the different characteristics listed by Bateson in his attempt to work towards a definition of "high" we have: "A 'high' civilization should therefore be presumed to have, on the technological side, whatever gadgets are necessary to promote, maintain (and even increase) wisdom of this 
general sort. This may well include computers and complex communication devices" (Bateson, 1972) (see also Bruni, 2001).

Such a direction would not only imply the "... optimization of the technosphere in order to reduce the burden on the environment" (Hofstetter, 1998), but also the educational and persuasion process for intervening culturally in the "hubris" loop illustrated by Bateson. In this sense the new emerging field of "persuasive technology" seems to be of interest here (see Fogg (1999), Berdichevsky and Neuenshwander (1999), Cornelissen et.al. (2006)), as long as the "persuasion" embedded in technology does not become phagocytized by the "hubris" and actually addresses the many unsustainable patterns continuously reinforced in the cultural positive feedbacks. Similar proposals come from the field of "Industrial Ecology" (Frosch and Gallopoulos, 1989) which uses natural ecological systems as a model for the design of sustainable industrial and technological systems. In order to do so, instead of focusing exclusively on material and energy flows (that by analogy are referred to as "industrial metabolism") this approaches also have an emphasis on "information flow" which is equally important in ecosystems and especially in human social and cultural life.

In the light of the tendencies of the "technospheric" digital expansion we should expect the development of a more "intelligent" technosphere in two different but relatable connotations:

- Intelligent in the sense of being adaptive, responsive, interactive and context-aware. This would be for example the direction of initiatives such as "ambient intelligence", the "Internet of things" or the "semantic web", which are however not necessarily related to sustainability concepts.

- Intelligent in the sense of encouraging, persuading and implementing sustainability and social responsibility.

The development of a "pervasive-intelligent" technosphere should therefore include, as much as possible, considerations about the persuasive potential for sustainability already from the outset of its very design, i.e.: by affording and encouraging sustainable behaviors and livelihoods, instead of waiting for the infrastructure to be deployed and then attempt to design a rhetoric strategy on top of it.

How much these two connotations of "intelligence" are effectively combined will be the core ethical challenges for the designers and engineers of future platforms, networks and applications and for users in general.

\section{Persuasion for Sustainability}

To summarize, in the present description, the technosphere is represented by the immense global infrastructure for communication, experience and action made possible by digital-immersiveinteractive-representational technologies. On the other hand, the semiosphere represents the virtual extension of the dynamic cultural "landscape" (or semiotic space) that is reachable, actualisable and potentially experienced by a human cognizer through such technological platform. Lastly, all these is happening and can only happen embedded in the biosphere and its life-support systems without which no cybernaut stands a chance for survival (Figure 3). 


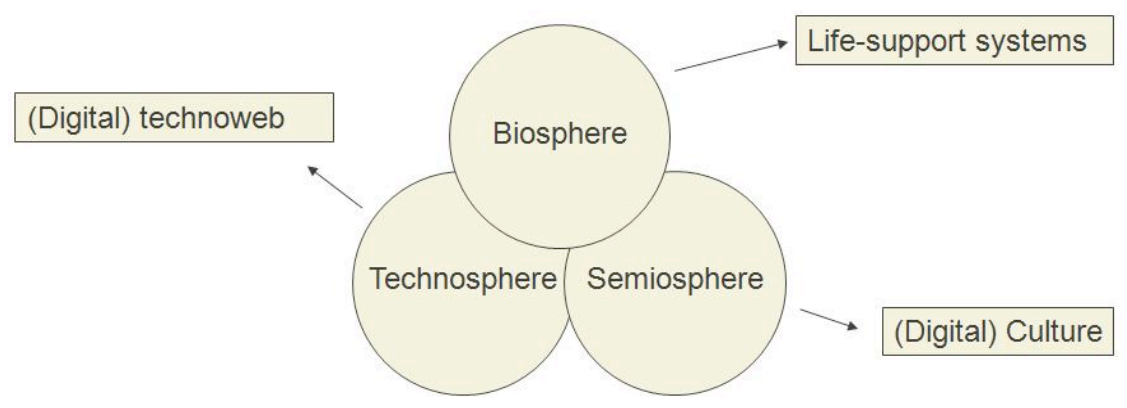

Figure 3: Man and Woman in the Biosphere, the Semiosphere and the Technosphere ${ }^{1}$

What sort of old and new, bad and good, epistemological and cultural habits are being shaped and re-shaped in such a semiosphere?

What are the cultural tendencies and currents that the efforts for encouraging sustainability will continuously encounter and which will work in counter-action in the realm of digital culture?

In this sense one of the main challenges will be that of implementing sustainable strategies in the so-called "experience economies", "cognitive and cultural economies", "attention economies" and the likes, where by means of information smog and overflow the real scarce resource is precisely the people's attention, with new forms of cultural franchising and whole industrial sectors relying "on human cognition, sensibility and social intelligence and their enhanced operation by means of digital information and communication technologies ... adding distinctive aesthetic and semiotic content to otherwise exclusively utilitarian goods (and services)" (Lorenzen et.al., 2008).

With the expansion of these trends of cultural/creative/cognitive/experiential industries, what new problems of inter-cultural communication, cultural homogenization and self-validating epistemological errors and unsustainable behaviors can we expect?

Even though there is obviously no possibility for designing top-down grand-systems for sustainability in sophisticated techno-webs (there would also be questions of eco-totalitarianism here), any bottom-up initiative needs probably to be concerted in some sort of collective strategy or framework in order to have a chance of making a difference.

Some important questions, within the technosphere-semiosphere relation and the resulting "digital culture" are:

- To start with, how to introduce persuasive elements for sustainability through the already existing infrastructure of the technosphere?

- How to prioritize values, objectives and "the object of persuasion", i.e.: the "content" of the digital world and governance in its semiosphere?

- How do we link these processes to the on-going debate that tries to discern how to define what is sustainable, what is socially responsible and what is ethical in general?

While the pervasiveness of sophisticated intelligent, automated, immersive, interactive, virtual or augmented environments may be an increasing trend in the future of the industrialized parts of the world it will still for long only cover an elite part of the world population (in spite of the populist dreams that may claim the contrary). Here lies the paradox of whether total global inclusion would actually determine an unsustainable development of the technosphere, in terms of energy and

\footnotetext{
${ }^{1}$ The Biosphere, consisting of the global ecological system integrating all living beings and their relationships, includes human beings with their cultural and technological developments. The technosphere has grown from the mere "builtenvironment" to the digital techno-web. The interconnectivity through the technosphere has increased the possibilities for cultural exchange in the global virtual space of the Semiosphere. Such cultural process is a central element in the determination of sustainable paths for men and woman in the biosphere and its life-support systems, without which human civilization cannot exist.
} 
material consumption for its design, construction and maintenance, not to mention the potentiality of potentiating positive feedback loops of unsustainable cultural processes (e.g. consumerism, speculation, slavery, prostitution, violence, exploitation, etc.) in the concomitant enlargement of the semiosphere, if there are not enough and effective efforts to counteract such tendencies.

Even though the percentage of the world population connected to digital culture is still a minority, it is in digital culture where unsustainable cultural premises and tendencies are potentiated the most.

\section{Conclusions}

The digital revolution has set in motion an irreversible development of a huge technosphere which by the principle that "technology brings more technology" (in a sort of positive feedback) should be expected to continue a rapid evolution and expansion around the whole planet with increase in connectivity, pervasiveness and "immersiveness". To these developments we need to add the advent of so-called mind and cognitive technologies and the promises and perils of the new "neurocivilization" (see Rose, 2005).

Independently of whether these tendencies will head towards utopic or dystopic directions there will be many ethical concerns and new challenges. It is hard to say which combination of the many societies and economies that have been postulated in relation to these developments will actually prevail: Information Society, Hypercomplex Society, Global Village, Digital Society, Knowledge Society, Network Society, Risk Society, Communication Society, Media Society, Experience Economy, Attention Economy, Learning Economy ... It will most likely be a combination of many of these characterizations.

There will be a myriad of contradictory cultural tendencies mingling in the enlarged semiosphere that the technosphere facilitates. The massive development of such technosphere can be a huge asset for sustainability or can reveal itself as one of the main factors or channels for what may become some of the worse forms of pollution ever produced by Modernity: cognitive dissonance, information overflow, media hyper-consumption, attention deficit and fake dematerialization of the economy, which all encourage alienation from the life-support systems of the biosphere, anxiety, violence, a state of social and economic disarray and a generalized divorce from reality.

From many scientific and philosophical areas we hear voices that warn us that the technical and technological advances are running much faster than their ethical understanding. We are still under the influence of the 19th century's positivistic idea that history is a process of continuous and irreversible progress. But we cannot refer to progress in everything indistinctly but to one or other particular field. In the same manner that there can be progress, regress can also take place. Sometimes both things can happen simultaneously in different fields, e.g. in the technological-material field and in the ethical field. Sometimes we speak also of "intellectual progress", which we often treat as synonym of "scientific progress", and in turn the latter is equated to experimental sciences and its industrial and technological applications. In this way intelligence is "degraded" by identifying it exclusively with the action of manipulating matter for practical purposes. Therefore by a transitive relation "intellectual progress" = "material progress".

The ethical challenge for the future of information and communication technology in this context is not to become an autotelic activity in which the goal is the use of technology itself. The challenge lies in how to deal with the "content" in the many cultural currents overlapping in the semiosphere, and, as in any ethical endeavor, how to define the ontological sources of legitimacy for sustainability, social responsibility and respect for cultural diversity.

With the increase of connectivity and the overflow of information we tend to believe that we are moving from information society to knowledge society. This optimistic view takes for granted that quantity per se will guarantee the qualitative jump from information to knowledge. Paraphrasing T.S. Elliot we can conclude by asking: "Where is the knowledge we have lost in information? Where is the wisdom we have lost in knowledge?" and where will the sources of wisdom come from in the future semiosphere? 


\section{References}

Bateson, G. (1972). Steps to an Ecology of Mind. New York: Chandler Publishing Company.

Batisse, M. (1973). Environmental Problems and the Scientists. Science and Public Affairs Bulletin of the Atomic Scientists, February.

Berdichevsky, D., and Neuenshwander, E. (1999). Toward an Ethics of Persuasive Technology. Communication of the ACM, 42(5), 51-58.

Blok, J. (2005). Environmental Exposure of Road Borders to Zinc. Science of the Total Environment, 348, 173-190.

Bruni, L. E. (2001). Biosemiotics and Ecological Monitoring. In W. Nöth and K. Kull (Ed.), Special Issue on Semiotics of Nature, Sign Systems Studies, 29(1). Tartu: Tartu University Press.

Cornelissen, G., Pandelaere, M., \& Warlop, L. (2006). Cueing Common Ecological Behaviors to Increase Environmental Attitudes. Persuasive Technology, 39-44.

Fogg, B.J. (1999). Persuasive Technologies. Communications of the ACM, 27-29.

Frosch, R.A. \& Gallopoulos, N.E. (1989). Strategies for Manufacturing. Scientific American 261(3): 144-152.

Hoc, J.-M. (2008). Cognitive Ergonomics: A Multidisciplinary Venture. Ergonomics, 51(1), 71-75.

Hofstetter, P. (1998). Perspectives in Life Cycle Impact Assessment: A Structured Approach to Combine Models of the Technosphere, Ecosphere and Valuesphere. USA: Kluwer Academic Publishers.

Jonas, H. (1984). The Imperative of Responsibility: In Search of an Ethics for the Technological Age. Chicago: The University of Chicago Press.

Lorenzen, M., Scott A. J. \& Vang, J. (2008). Editorial: Geography and the Cultural Economy. Journal of Economic Geography 8, 589-592.

Lotman, J. (2005/1984). On the Semiosphere. Sign Systems Studies 33(1).

Lotman, Y. (1990). Universe of the Mind: A Semiotic Theory of Culture. Bloomington: Indiana University Press.

Mueller, M. P. (2009). Educational Reflections on the "Ecological Crisis": EcoJustice, Environmentalism, and Sustainability. Sci \& Educ 18, 1031-1056.

Rose, S. (2005). The Future of the Brain: The Promise and Perils of Tomorrow's Neuroscience. Oxford: Oxford University Press.

Torop, P. (2005). Semiosphere and/as the Research Object of Semiotics of Culture. Sign Systems Studies 33(1).

\section{About the Author}

Luis Emilio Bruni

Born in 1963 in Caracas - Venezuela. Ph.D. in Molecular Biology and Theory of Science at the Institute of Molecular Biology, University of Copenhagen, Denmark. Master of Science in International and Global Relations at the Universidad Central de Venezuela. Bachelor of Science in Environmental Engineering at the Pennsylvania State University, USA. Since 2004 is at Aalborg University (Denmark) where he is associate professor at the Department of Architecture, Design and Media Technology and where he teaches courses on multimodal perception and cognition, digital culture and theory of science. His current research covers relations between cognition, technology and culture with focus on sustainability. For several years was guest lecturer in bioethics and sustainability at the Department of Biology of the University of Copenhagen. From 1987 to 1991 was researcher at the Communication Science and Artificial Intelligence Research Centre "Semeion" of Rome, Italy, where he started his research in semiotics, cybernetics and epistemological perspectives in culture-nature relations. From 1996 to 1999 served as especial adviser on biodiversity, bioethics and sustainable development to the Amazonian Parliament and was researcher at the Venezuelan Centre for Global and International Relations of the Universidad Central de Venezuela. Was founder member of the International Society for Biosemiotic Studies (ISBS), currently serves in its executive committee and is member of the editorial boards of the Biosemiotics Journal (Springer). 